\title{
USO DA TEORIA DO CONFORTO DE KOLCABA NA IMPLEMENTAÇÃO DO PROCESSO DE ENFERMAGEM: REVISÃO INTEGRATIVA
}

\section{USE OF THE THEORY OF KOLCABA COMFORT IN THE IMPLEMENTATION OF THE NURSING PROCESS: INTEGRATIVE REVIEW}

\section{USO DE LA TEORÍA DEL CONFORT DE KOLCABA EN LA IMPLEMENTACIÓN DEL PROCEDIMIENTO DE ENFERMADO: REVISIÓN INTEGRATIVA}

\author{
Rosane Barreto Cardoso ${ }^{1}$, Célia Pereira Caldas ${ }^{2}$, Priscilla Alfradique de Souza ${ }^{3}$
}

\begin{abstract}
RESUMO
Objetivo: Analisar as evidências científicas acerca do uso da teoria do conforto de Kolcaba na implementação do processo de enfermagem. Método: Trata-se de uma revisão integrativa, com estudos entre 2000 a 2017, com pesquisas realizada nos bancos de dados da CINAHL, LILACS, PubMED, SCOPUS e Web of Science, obedecendo seis etapas correlacionadas. Resultados: Foram encontrados 16 artigos dos quais emergiram quatro categorias temáticas: processo de enfermagem no contexto da saúde da criança, do adulto, da mulher e do idoso. Observou-se que o uso da teoria do conforto possibilitou aos enfermeiros cumprimento de fases do processo de enfermagem. Conclusão: Conclui-se que a teoria do conforto é um referencial teórico que fundamenta o processo de enfermagem e potencializa o conforto como resultado da assistência de enfermagem.
\end{abstract}

Descritores: Teoria de Enfermagem; Processo de Enfermagem; Conforto do Paciente.

\begin{abstract}
Objective: To analyze the scientific evidence about the use of Kolcaba comfort theory in the implementation of the nursing process. Method: It is an integrative review, with studies between 2000 and 2017, with researches done in the databases of CINAHL, LILACS, PubMED, SCOPUS and Web of Science, obeying six correlated steps. Results: There were 16 articles from which emerged four thematic categories: nursing process in the context of the health of the child, the adult, the woman and the elderly. It was observed that the use of comfort theory enabled nurses to comply with phases of the nursing process. Conclusion: It is concluded that the theory of comfort is a theoretical reference that bases the nursing process and enhances comfort as a result of nursing care.
\end{abstract}

Descriptors: Nursing Theory; Nursing Process; Patient Comfort.

\footnotetext{
${ }^{1}$ Mestre em Ensino em Ciências da Saúde. Pós-graduada em Planejamento, Implementação; Gestão da EAD e Informática em Saúde; Docência para o Ensino Superior em Enfermagem; Gestão em Enfermagem. Especialista em Enfermagem em Cardiologia. Graduada em Enfermagem. Enfermeira de Desenvolvimento Operacional e Administrativo, no Hospital Unimed Rio.

${ }^{2}$ Enfermeira. Pós-doutorado em Gerontologia pela Universidade de Jönköping, Suécia. Docente do Departamento de Enfermagem da Universidade Estadual do Rio de Janeiro.

${ }^{3}$ Enfermeira. Doutora em Enfermagem pela School of Nursing of University of Texas Health Science Center at San Antonio. Docente do Departamento de Enfermagem da Universidade Estadual do Rio de Janeiro.
} 


\section{RESUMEN}

Objetivo: Analizar las evidencias científicas acerca del uso de la teoría del confort de Kolcaba en la implementación del proceso de enfermería. Método: Se trata de una revisión integrativa, con estudios entre 2000 a 2017, con investigaciones realizadas en los bancos de datos de CINAHL, LILACS, PubMED, SCOPUS y Web of Science, obedeciendo seis etapas correlacionadas. Resultados: Se encontraron 16 artículos de los cuales surgieron cuatro categorías temáticas: proceso de enfermería en el contexto de la salud del niño, del adulto, de la mujer y del anciano. Se observó que el uso de la teoría del confort posibilitó a los enfermeros cumplimiento de fases del proceso de enfermería. Conclusión: Se concluye que la teoría del confort es un referencial teórico que fundamenta el proceso de enfermería y potencia el confort como resultado de la asistencia de enfermería.

Descriptores: Teoría de Enfermería; Proceso de Enfermería; Comodidad del Paciente.

\section{INTRODUÇÃO}

A Sistematização da Assistência de Enfermagem (SAE) provê elementos para a organização e gerenciamento do cuidado, o que possibilita a implementação do Processo de Enfermagem (PE). ${ }^{1-2} \mathrm{O}$ PE é um instrumento metodológico que requer incorporação de perspectivas de teorias de enfermagem para tornar os resultados da assistência operacionalizáveis. $^{2}$

As teorias de enfermagem estabelecem a base do conhecimento científico para sistematizar o saber e organizar o cuidado, o que institui subsídios para uma prática profissional baseada em evidências. Pode ser entendida como um conjunto específico e concreto de conceitos e proposições para explicar, descrever, prever ou caracterizar fenômenos de interesse à disciplina de enfermagem. ${ }^{3-5}$

Observa-se na prática uma lacuna de suporte teórico para determinação dos cuidados, essencialmente para a operacionalização do PE com enfoque na promoção do conforto, como ressaltado na teoria de Katharine Kolcaba.

O conforto é considerado como uma necessidade básica da pessoa humana, um resultado essencial do cuidado de enfermagem, universalmente desejável, relevante em várias taxonomias profissionais e teorias de enfermagem. ${ }^{6-8}$

Na teoria de Kolcaba o conforto é ressaltado como uma experiência imediata, fortalecida por sensação de alívio, tranquilidade e transcendência, considerando o contexto físico, psicoespiritual, sociocultural e ambiental. ${ }^{7}$

Mediante ao exposto considera-se relevante contextualizar e analisar a teoria do conforto de Kolcaba, com o intuito de conhecer sua aplicabilidade na implementação do PE.

Desse modo por reconhecer a importância da fundamentação teórica enquanto ciência, organização e qualidade 
da assistência, tem-se como objetivo desta pesquisa analisar artigos científicos publicados no cenário mundial acerca do uso da teoria do conforto de Kolcaba na implementação do PE.

Este estudo visa fomentar a prática de enfermagem baseada em evidências, bem como proporcionar subsídios para o planejamento adequado do PE.

\section{METODOLOGIA}

Trata-se de uma revisão integrativa (RI) da literatura, a qual sintetiza os estudos disponíveis sobre determinado tema e conduz a prática baseando-se em conhecimento cientifico. ${ }^{9-10}$ Tem como proposta gerar conhecimento sobre um problema e determinar se sua aplicação é viável na prática. ${ }^{10}$ Este método possibilita a síntese de múltiplos estudos publicados, muito útil para enfermeiros que estão na prática clínica e desejam realizar assistência de enfermagem baseada em evidências científicas. $^{11}$

Para construção desta RI, trilhou-se um percurso metodológico que contemplou seis etapas: $1^{\text {a }}$ ) Identificação do tema e seleção da questão de pesquisa; $2^{a}$ ) Estabelecimento dos critérios de inclusão e exclusão; $3^{\mathrm{a}}$ ) Identificação dos artigos pré-selecionados e selecionados; $4^{\mathrm{a}}$ ) Categorização dos artigos; $5^{\text {a) }}$ Construção da análise dos resultados; e $\left.6^{a}\right)$ Apresentação da síntese do conhecimento. $^{12}$
A primeira etapa da RI consistiu-se na identificação do tema e seleção da questão norteadora, que versou em: Qual é a produção científica acerca da aplicação da teoria do conforto de Kolcaba na implementação do PE?

$\mathrm{Na} 2^{\mathrm{a}}$ etapa, estipulou-se como critérios de inclusão: estudos que abordassem o uso da teoria do conforto de Kolcaba na implementação do processo de enfermagem; que se encontrassem disponíveis na íntegra sob a modalidade de artigo científico; publicado entre 2000 a 2017, na língua portuguesa, inglesa e espanhola. De acordo com os critérios de exclusão foram excluídos artigos repetidos, de revisões e aqueles que após a leitura na íntegra não responderam à questão de pesquisa.

A $3^{\text {a }}$ etapa consistiu-se na identificação dos artigos pré-selecionados durante a coleta de dados. A busca foi realizada no decorrer dos meses de abril e maio de 2017, nas seguintes bases de dados: Cumulative Index to Nursing and Allied Health Literature (CINAHL), Literatura Latino Americana e do Caribe em Ciências da Saúde (LILACS), United Station National Library of Medicine (PubMED), SciVerse Scopus (SCOPUS) e Web Of Science.

A escolha das bases de dados foi devido ao quantitativo de indexação de artigos da área da saúde, bem como temáticas relacionadas à enfermagem. Foram 
utilizados os seguintes descritores em ciências da saúde: teoria de enfermagem, processo de enfermagem e conforto do paciente, nos idiomas português, inglês e espanhol de acordo com a base de dados, utilizando os operadores booleanos AND e OR para cruzamento, conforme apresentado no Quadro 1.

Quadro 1. Estratégia de busca e determinação da amostra em cada base de dados. Rio de Janeiro, Brasil, 1994-2017.

\begin{tabular}{|c|c|c|c|c|c|}
\hline $\begin{array}{r}\text { Bases } \\
\text { de Dados }\end{array}$ & 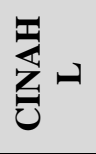 & $\underset{\exists}{J}$ & $\sum_{0}^{\infty} 0$ & $\bigodot_{\mathscr{n}}^{\ominus} \sim$ & 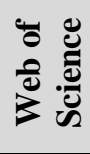 \\
\hline $\begin{array}{c}\text { Teoria de enfermagem/Nursing theor/Teoría de enfermería } \\
\text { OR Processo de enfermagem/Nursing Process/Proceso de } \\
\text { Enfermería }\end{array}$ & 7502 & 734 & 6086 & 5129 & 856 \\
\hline $\begin{array}{c}\text { Conforto do paciente/Patient comfort/Comodidade del } \\
\text { paciente OR Processo de enfermagem/Nursing } \\
\text { Process/Proceso de Enfermería }\end{array}$ & 2079 & 324 & 3390 & 4506 & 2545 \\
\hline $\begin{array}{l}\text { Teoria de enfermagem/Nursing theory/Teoría de enfermeira } \\
\text { AND Conforto do paciente/Patient comfort/Comodidade del } \\
\text { paciente }\end{array}$ & 20 & 8 & 102 & 95 & 72 \\
\hline Artigos pré-selecionados & 11 & 6 & 10 & 9 & 5 \\
\hline Artigos repetidos & - & 3 & 6 & 8 & 5 \\
\hline Artigos selecionados & 9 & 3 & 3 & 1 & $\mathbf{0}$ \\
\hline
\end{tabular}

Fonte: Autores, 2017.

Após o procedimento de busca as publicações pré-selecionadas foram localizadas com base na leitura do título e resumo. Após a leitura na íntegra das publicações previamente selecionadas $(n=41)$, a amostra final de artigos foi definida $(n=16)$.

A $4^{\text {a }}$ etapa da revisão integrativa consistiu-se na categorização dos artigos selecionados. A extração dos dados foi realizada por meio de um roteiro semiestruturado elaborado pelos autores. $\mathrm{O}$ roteiro não foi validado, mas possibilitou a extração de informações dos estudos selecionados, como: título do artigo, autores, ano de publicação, idioma, revista de publicação, objetivo, metodologia, população e cenário do estudo, resultados e conclusões.

A $5^{\text {a }}$ etapa incidiu-se na construção da análise dos resultados. Optou-se por realizar análise descritiva simples apresentando-as em categorias. A $6^{\text {a }}$ etapa consistiu na apresentação da síntese do conhecimento, que foi apresentada nos próximos tópicos desta RI.

Por se tratar de uma RI, não foi necessário solicitar aprovação do Comitê de Ética para realização do estudo. 


\section{RESULTADOS E DISCUSSÃO}

Nesta RI foram analisados 16 artigos originais que atenderam aos critérios de inclusão previamente estabelecidos. Dentre os estudos, $12,5 \%$ (2) foram publicados em 2000, 6,2\% (1) em 2002, um em 2004, um em 2005, um em 2012, 25\%
(4) em 2014, dois em 2015, 18,7\% (3) em 2016 e um em 2017. Em relação à origem dos estudos, $56,2 \%$ (9) foram publicados no idioma inglês e em periódicos internacionais, e 43,7\% (7) no idioma português, conforme apresentado no quadro 2.

Quadro 2 - Relação de artigos inclusos no estudo (E). Rio de Janeiro, Brasil, 2017.

\begin{tabular}{|c|c|c|c|}
\hline $\mathbf{E}$ & Título/ Autor & $\begin{array}{l}\text { Idioma } \\
\text { Ano }\end{array}$ & Periódico \\
\hline \begin{tabular}{l|}
$\mathrm{E}$ \\
1
\end{tabular} & $\begin{array}{l}\text { Acute care for elders (ACE): a holistic model for geriatric } \\
\text { orthopaedic nursing care. }{ }^{13} \text { Panno } \mathrm{J} \mathrm{M} \text {, Kolcaba } \mathrm{K} \text {, Holder } \mathrm{C}\end{array}$ & $\begin{array}{c}\text { Inglês } \\
2000\end{array}$ & Orthop Nurs. \\
\hline $\begin{array}{l}\mathrm{E} \\
2\end{array}$ & $\begin{array}{l}\text { Alternative and complementary therapies for labor and birth: an } \\
\text { application of Kolcaba's theory of holistic comfort. }{ }^{14} \text { Koehn ML }\end{array}$ & $\begin{array}{l}\text { Inglês } \\
2000\end{array}$ & $\begin{array}{l}\text { Holist Nurs } \\
\text { Pract }\end{array}$ \\
\hline $\begin{array}{l}\mathrm{E} \\
3\end{array}$ & $\begin{array}{l}\text { Comfort care: a framework for perianesthesia nursing. }{ }^{15} \text { Kolcaba K, } \\
\text { Wilson L }\end{array}$ & $\begin{array}{c}\text { Inglês } \\
2002\end{array}$ & $\begin{array}{l}\text { J Perianesth } \\
\text { Nurs. }\end{array}$ \\
\hline $\begin{array}{l}\mathrm{E} \\
4\end{array}$ & $\begin{array}{l}\text { Practical application of comfort theory in the perianesthesia setting. }{ }^{16} \\
\text { Wilson L, Kolcaba K }\end{array}$ & $\begin{array}{l}\text { Inglês } \\
2004\end{array}$ & $\begin{array}{l}\text { J Perianesth } \\
\text { Nurs }\end{array}$ \\
\hline $\begin{array}{l}\mathrm{E} \\
5\end{array}$ & $\begin{array}{l}\text { Comfort theory and its application to pediatric nursing. }{ }^{17} \text { Kolcaba K, } \\
\text { DiMarco MA }\end{array}$ & $\begin{array}{l}\text { Inglês } \\
2005\end{array}$ & Pediatr Nurs. \\
\hline $\begin{array}{l}\mathrm{E} \\
6\end{array}$ & $\begin{array}{l}\text { Contribuição do cuidado clínico de enfermagem para o conforto } \\
\text { psicoespiritual de mulheres infarto agudo do miocárdio(IAM). }{ }^{18} \\
\text { Ponte KMA, Silva LF, Aragão AEA, Guedes MVC, Zagonel IPS }\end{array}$ & $\begin{array}{l}\text { Português } \\
2012\end{array}$ & $\begin{array}{l}\text { Esc. Anna } \\
\text { Nery }\end{array}$ \\
\hline $\begin{array}{l}\mathrm{E} \\
7\end{array}$ & $\begin{array}{l}\text { Cuidados de enfermagem a uma puérpera fundamentados na teoria } \\
\text { do conforto. }{ }^{19} \text { Barbosa EMG, Oliveira FDM, Guedes MVC, } \\
\text { Monteiro ARM, Rodrigues DP, Silva LF, Fialho AVM. }\end{array}$ & $\begin{array}{l}\text { Português } \\
2014\end{array}$ & $\begin{array}{l}\text { Rev Min } \\
\text { Enferm. }\end{array}$ \\
\hline $\begin{array}{l}\mathrm{E} \\
8\end{array}$ & $\begin{array}{l}\text { A practical application of Katharine Kolcaba's comfort theory to } \\
\text { cardiac patients. }{ }^{20} \text { Krinsky R, Murillo I, Johnson J. }\end{array}$ & $\begin{array}{l}\text { Inglês } \\
2014\end{array}$ & Appl Nurs Res \\
\hline $\begin{array}{l}\mathrm{E} \\
9\end{array}$ & $\begin{array}{l}\text { End-of-life care in the neonatal intensive care unit: applying comfort } \\
\text { theory. }{ }^{21} \text { Marchuk A. }\end{array}$ & $\begin{array}{l}\text { Inglês } \\
2016\end{array}$ & J Nurs Palliat \\
\hline $\begin{array}{l}\mathrm{E} \\
1 \\
0\end{array}$ & $\begin{array}{l}\text { Cuidados de enfermagem a mulheres com infarto do miocárdio: } \\
\text { promoção do conforto sociocultural pela pesquisa-cuidado. }{ }^{22} \text { Ponte } \\
\text { KMA, Silva LF. }\end{array}$ & $\begin{array}{l}\text { Português } \\
2014\end{array}$ & $\begin{array}{l}\text { Rev enferm } \\
\text { UERJ }\end{array}$ \\
\hline $\begin{array}{l}\mathrm{E} \\
1 \\
1\end{array}$ & $\begin{array}{l}\text { Cuidado clínico de enfermagem para conforto de mulheres com } \\
\text { IAM. }{ }^{23} \text { Ponte KMA, Silva LF, Aragão AEA, Guedes MVC, Zagonel } \\
\text { IPS. }\end{array}$ & $\begin{array}{l}\text { Português } \\
2014\end{array}$ & $\begin{array}{l}\text { Texto } \\
\text { Contexto } \\
\text { Enferm }\end{array}$ \\
\hline $\begin{array}{l}\mathrm{E} \\
1 \\
2\end{array}$ & $\begin{array}{l}\text { Processo de enfermagem no conforto do paciente com insuficiência } \\
\text { cardíaca (IC) no domicílio. }{ }^{24} \text { Freire da Silva FV, Rabelo ACS, Silva } \\
\text { LF. }\end{array}$ & $\begin{array}{l}\text { Português } \\
2015\end{array}$ & $\begin{array}{l}\text { Aquichan } \\
\text { LILACS }\end{array}$ \\
\hline $\begin{array}{l}\mathrm{E} \\
1 \\
3\end{array}$ & $\begin{array}{l}\text { The Effect of Nursing Care Based on Comfort Theory on Women's } \\
\text { Postpartum Comfort Levels After Caesarean Sections. }{ }^{25} \text { Aksoy } \\
\text { Derya Y, Pasinlioglu T. }\end{array}$ & $\begin{array}{l}\text { Inglês } \\
2015\end{array}$ & $\begin{array}{l}\text { Int J Nurs } \\
\text { Knowl }\end{array}$ \\
\hline \begin{tabular}{l|} 
E \\
1 \\
4
\end{tabular} & $\begin{array}{l}\text { Utilidade da teoria do conforto para o cuidado clínico de enfermagem } \\
\text { à puérpera: análise crítica. }{ }^{26} \text { Lima JVF, Guedes MVC, Silva LF, } \\
\text { Freitas MC, Fialho AVM }\end{array}$ & $\begin{array}{l}\text { Português } \\
2016\end{array}$ & $\begin{array}{l}\text { Rev Gaúcha } \\
\text { Enferm }\end{array}$ \\
\hline
\end{tabular}




\begin{tabular}{|l|l|c|l|}
\hline E & $\begin{array}{l}\text { Epilepsy monitoring - The patients' views: A qualitative study based } \\
\text { on Kolcaba's Comfort Theory. }{ }^{27} \text { Egger-Rainer A, Trinka E, Höfler J, } \\
5\end{array}$ & $\begin{array}{c}\text { Inglês } \\
2017\end{array}$ & $\begin{array}{l}\text { Epilepsy } \\
\text { Behav }\end{array}$ \\
\hline E & $\begin{array}{l}\text { Comfort Theory in Practice-Nurse Anesthetists' Comfort Measures } \\
1\end{array}$ & $\begin{array}{l}\text { Inglês } \\
\text { and Interventions in a Preoperative Context. }{ }^{28} \text { Bergström A, A }\end{array}$ & $\begin{array}{l}\text { J Perianesth } \\
\text { Nurs }\end{array}$ \\
\hline
\end{tabular}

Fonte: Autores, 2017.

A amostra foi composta por produções internacionais e nacionais, validando acentuada influência global da promoção do conforto como resultado da assistência de enfermagem.

Em relação à abordagem dos estudos selecionados, $87,5 \%$ (14) foram qualitativos e $12,5 \%$ (2) foram quantitativos (um ensaio clínico controlado e um estudo quase experimental). Dentre as pesquisas qualitativas cinco foram estudos de caso, cinco estudos descritivos, três estudos pesquisa-cuidado e um estudo teórico reflexivo.

A população alvo dos estudos foi composta por idosos, crianças, puérpera, mulheres em trabalho de parto, mulheres com diagnóstico médico de infarto agudo do miocárdio (IAM), pacientes adultos com insuficiência cardíaca (IC), síndrome coronariana aguda (SCA) e epilepsia.

A análise dos artigos permitiu compor as seguintes categorias temáticas, representadas por: processo de enfermagem no contexto da saúde da criança, do adulto, da mulher e do idoso.

A primeira categoria temática denominada "processo de enfermagem no contexto da saúde da criança" foi constituída por dois artigos (E5 e E9). Nesta categoria foi evidenciada a aplicação da teoria do conforto por enfermeiros para promoção da saúde da criança.

Kolcaba e DiMarco ${ }^{17}$ relataram o uso da estrutura taxonômica do conforto (ETC) como instrumento de coleta de dados e o desenvolvimento de um questionário para avaliação do conforto da criança no ambiente hospitalar. Maechuk ${ }^{21}$ descreveu em seu estudo o uso da ETC por enfermeiras e parteiras para implementação do $\mathrm{PE}$ na assistência à recém-nascidos em cuidados paliativos.

$\mathrm{Na}$ segunda categoria "processo de enfermagem da saúde da mulher" foram classificados sete artigos (E2, E6, E7, E10, E11, E13 e E14). Nesta categoria foi identificada a aplicação da teoria do conforto na sistematização da assistência de enfermagem a mulheres.

Koehn ${ }^{14}$ abordou o uso da teoria do conforto como guia para enfermeiros na implementação de cuidados baseados em terapias alternativas e complementares (musicoterapia, acupuntura, aromaterapia, hidromassagem e massoterapia) na 
assistência a mulheres em trabalho de parto e seus filhos recém-nascidos.

Aksoy Derya e Pasinlioglu ${ }^{25}$ elaboraram um livreto que foi utilizado como referencial para treinamento de enfermeiros quanto à implementação do PE na assistência a mulheres no período do pósparto.

Barbosa et $\mathrm{al}^{19}$, Aksoy Derya e Pasinlioglu ${ }^{25}$ demostraram em seus estudos a aplicação da teoria do conforto no cuidado sistematizado de enfermagem conduzidos à puérperas. Nestes estudos foram mencionados alguns diagnósticos de enfermagem estratificados por dimensões do conforto, tais como:

- Contexto físico: risco de sangramento; dor aguda, náusea; risco de infecção; risco de integridade da pele prejudicada; risco de trauma vascular; retenção urinária; risco de constipação; disposição para nutrição melhorada; volume de líquidos deficiente; privação de sono; fadiga; mobilidade física prejudicada; disposição para o controle da saúde melhora; e amamentação ineficaz. 19,25

- Contexto psicoespiritual: ansiedade; risco de solidão; baixa autoestima situacional; distúrbio da imagem corporal; tensão do papel do cuidador; processos familiares disfuncionais; e disfunção sexual. 19,25

- Contexto sociocultural: conhecimento deficiente. ${ }^{19,25}$

- Contexto ambiental: conforto prejudicado. ${ }^{19,25}$

Ponte et al ${ }^{18,22-23}$ publicou três artigos brasileiros recortes de sua tese de doutorado, que abordaram a interação do método pesquisa-cuidado e a aplicação da teoria do conforto a mulheres hospitalizadas. A autora analisou a contribuição do cuidado de enfermagem mediado por tecnologias na promoção do conforto físico, psicoespiritual, sociocultural e ambiental, ofertados a nove mulheres com diagnóstico médico de IAM.

Lima et $\mathrm{al}^{26}$ avaliou a aplicação do conceito da teoria do conforto para a implementação do $\mathrm{PE}$ na promoção do conforto puerperal no ambiente hospitalar.

$\mathrm{Na}$ terceira categoria temática "processo de enfermagem no contexto da saúde do adulto" foram correlacionados seis artigos (E3, E4, E8, E12, E15 e E16). Nesta categoria foi observado o conforto como resultado da assistência de enfermagem ofertado à pacientes adultos.

Kolcaba e Wilson ${ }^{15-16}$, e Bergström et $\mathrm{al}^{28}$ discutiram em seus estudos o uso da ETC no contexto pré e peri-anestésico, e a elaboração de um questionário para 
avaliação do conforto de pacientes no período pré-anestésica.

Krinsky et $\mathrm{al}^{20}$ e Freire da Silva et $\mathrm{al}^{24}$ descreveram o uso da teoria do conforto na implementação do PE para a promoção do conforto à pacientes cardiopatas. EggerRainer et $\mathrm{al}^{27}$ demostrou a aplicação da teoria do conforto no cuidado à pacientes internados em uma unidade de monitoramento de epilepsia.

Na quarta categoria temática "processo de enfermagem no contexto da saúde do idoso" foi estratificado um artigo (E1). Nesta categoria evidenciou a aplicação da teoria do conforto na promoção da saúde do idoso. Panno et $\mathrm{al}^{13}$ abordou a aplicação de um modelo de cuidados geriátricos baseado nos princípios da teoria do conforto para o manejo clínico de pacientes idosos com problema ortopédicos.

Observa-se em todas as categorias temáticas que o uso da teoria do conforto possibilitou aos enfermeiros cumprimento da primeira fase do PE, a coleta de dados ou histórico de enfermagem. Tal fato favoreceu uma coleta de dados estruturada e direcionada a identificação das necessidades não satisfatórias e desconfortos dos pacientes. A coleta de dados fundamentada em uma teoria de enfermagem proporciona subsídios concretos para a execução das demais etapas do PE. ${ }^{1-2}$
Os artigos relacionados à categoria temática "processo de enfermagem no contexto da saúde da mulher" foram os que apresentaram realces de cumprimento da segunda etapa do PE, com a formulação dos diagnósticos de enfermagem. Quando baseados em evidências, os diagnósticos de enfermagem são elementos primordiais para obtenção de melhores resultados e direcionamento das intervenções de enfermagem. ${ }^{1-3}$

Foram evidenciadas nos artigos analisados algumas propostas de atividades para intervenções de enfermagem relacionadas à promoção do conforto físico, ambiental, psicoespiritual e sociocultural. Tal fato corrobora com as proposições da teórica em estudo, ao afirmar que quando as intervenções são realizadas de forma consistente podem promover ou facilitar comportamentos de busca em saúde. ${ }^{7}$

Também foi observado nas análises o empenho dos autores no desenvolvimento de questionários e escalas para mensuração do conforto dos pacientes. Tal prática pode estar relacionada à busca pela implementação da proposição teórica e sua correlação prática através de instrumentos norteadores da assistência. Além disso, durante a formação acadêmica, o uso dos instrumentos facilita o processo de ensino e aprendizado, o que pode ser uma excelente ferramenta na consolidação dos cuidados de enfermagem para promoção do conforto. 
O uso da teoria do conforto na implementação do PE, além de estruturar o cuidado, eleva o nível da assistência prestada, fortalece as relações enfermeiropaciente-família e favorece o desenvolvimento de comportamentos de busca em saúde, podendo aprimorar os resultados institucionais. ${ }^{13-28}$ Desta forma, espera-se que este estudo possa contribuir com o desenvolvimento de pesquisas que visem a promoção do conforto como cerne do cuidado do enfermeiro, tanto para intervenções com enfoque no conforto, quanto para a avaliação de resultados individuais e institucionais, que empreguem o conforto como indicador de qualidade da assistência.

Como contribuição para a prática clínica, os achados desta RI apoiaram a indicação do uso da teoria do conforto para implementação do PE, objetivando como resultado de enfermagem a promoção do conforto ao paciente nos contextos físico, psicoespiritual, ambiental e sociocultural.

\section{CONCLUSÃO}

Os resultados deste estudo foram limitados pela escassez de pesquisas que apresentassem a aplicação da teoria perpassando por todas as etapas do PE, assim como o predomínio de estudos descritivos e relatos de casos, o que influenciou a busca de achados com alto nível de evidência que pudessem ser utilizados para transposição na prática clínica.

A RI ratificou as contribuições do uso da teoria do conforto de Kolcaba para a implementação do PE em diferentes populações, sendo utilizada na promoção da saúde da criança, do adulto em especial mulheres, e saúde do idoso.

A produção do conhecimento promulgou o emprego da teoria do conforto na implementação do PE, vislumbrando o crescente interesse do conforto holístico como resultado de enfermagem, com proposta de melhoria da qualidade da assistência a partir da utilização de uma teoria de enfermagem.

A teoria do conforto apresentou-se como um referencial teórico que avigora a enfermagem enquanto ciência, além incentivar a interação, autonomia e valorização das necessidades do paciente a partir da promoção do conforto, com consequente melhoria da qualidade de vida.

\section{REFERENCIAS}

1. Conselho Federal de Enfermagem (Brasil). Resolução 358/2009, de 15 de outubro de 2009. Dispõe sobre a Sistematização da Assistência de Enfermagem e a implementação do Processo de Enfermagem em ambientes públicos ou privados, em que ocorre o cuidado profissional de Enfermagem. Brasília, DF: COFEN; 2009.

2. Fuly PSC, Leite JL, Lima SBS. Correntes de pensamento nacionais sobre rentes de pensamento nacionais 
sobre sistematização da assistência de enfermagem. Rev Bras Enferm.

[Internet]. 2008 [citado em 01 jan 2018]; 61(6):883-7.

doi:/10.1590/S003471672008000600015

3. Reis GS, Reppetto MA, Santos LSC, Devezas AMLO. Sistematização da assistência de enfermagem: vantagens e dificuldades na implantação. Arq Med Hosp Fac Ciênc Méd Santa Casa São Paulo. 2016; 61(n.esp).

4. McEwen M, Wills EM. Bases teóricas para enfermagem. 4ed. Porto Alegre: Artmed; 2016.

5. Schaurich D, Crossetti MGO. Produção do conhecimento sobre teorias de enfermagem: análise de periódicos da área - 1998-2007. Esc Anna Nery Rev Enferm. [Internet]. 2010 [citado em 01 jan 2018]; 14(1):182-88.

doi:/10.1590/S141481452010000100027

6. Silva EGC, Oliveira VC, Neves GBC, Guimarães TMR. O conhecimento do enfermeiro sobre a sistematização da assistência de enfermagem: da teoria à prática. Rev Esc enferm. [Internet]. 2011[citado em 01 jan 2018]; 45(6):1380-6. doi:/10.1590/S008062342011000600015

7. Kolcaba K. Comfort theory and practice: a vision for holistic health care and research. New York: Springer Publishing Company; 2003.

8. Apostolo JLA, Kolcaba K. The effects of guided imagery on comfort, depression, anxiety, and stress of psychiatric inpatients with depressive. Arch Psychiatr Nurs. [Internet]. 2009 [citado em 01 jan 2018]; 23(6):403-11. doi:/10.1016/j.apnu.2008.12.003

9. Ponte KMA, Silva LF. Conforto como resultado do cuidado de enfermagem: revisão integrativa. Rev Pesqui Cuid. Fundam. [Internet]. 2015 [citado em 01 jan 2018]; 7(3):2603-14. doi:/10.9789/21755361.2015.v7i2.2603-2614
10. Souza MT, Silva MD, Carvalho R. Integrative review: what is it? How to do it? Einstein. [Internet]. 2010 [citado em 01 jan 2018]; 8(1 Pt 1):102-6. doi:/ 10.1590/s1679-45082010rw1134

11. Crossetti MGO. Integrative review of nursing research: scientific rigor required. Rev Gaúch Enferm. [Internet]. 2012 [citado em 01 jan 2018]; 33(2):12-13. doi:/10.1590/S198314472012000200003

12. Soares CB, Hoga LAK, Peduzzi M, Sangaleti C, Yonekura T, Silva D. Integrative review: concepts and methods used in nursing. Rev Esc Enferm USP. [Internet]. 2014 [citado em 01 jan 2018]; 48(2):335-45. doi:/10.1590/S00806234201400002000020

13. Mendes KDS, Silveira RCCP, Galvão CM. Revisão integrativa: método de pesquisa para incorporação de evidências na saúde e na enfermagem. Texto \& Contexto Enferm. [Internet]. 2008 [citado em 01 jan 2018];17(4): 758-64. doi:/10.1590/S010407072008000400018

14. Panno J M, Kolcaba K, Holder C. Acute care for elders (ACE): a holistic model for geriatric orthopaedic nursing care. Orthop Nurs. 2000; 19(6):53-60.

15. Koehn ML. Alternative and complementary therapies for labor and birth: an application of Kolcaba's Theory of Holistic Comfort. Holist Nurs Pract. 2000; 15(1):66-77.

16. Kolcaba K, Wilson L. Comfort care: a framework for perianesthesia nursing. $\mathrm{J}$ Perianesth Nurs. [Internet]. 2002 [citado em 01 jan 2018]; 17(2):102-11. doi:/10.1053/jpan.2002.31657

17. Wilson L, Kolcaba K. Practical application of comfort theory in the perianesthesia setting. J Perianesth Nurs. [Internet]. 2004 [citado em 01 jan 2018]; 19(3):164-73. doi:/10.1016/j.jopan.2004.03.006

18. Kolcaba K, DiMarco MA. Comfort theory and its application to pediatric 
nursing. Pediatr Nurs. 2005; 31(3):187-94.

19. Ponte KMA, Silva LF, Aragão AEA, Guedes MVC, Zagonel IPS. Contribuição do cuidado clínico de enfermagem para o conforto psicoespiritual de mulheres com IAM.

Esc Anna Nery Rev Enferm. [Internet]. 2012 [citado em 01 jan 2018]; 16 (4):666-73. doi:/10.1590/S141481452012000400004

20. Barbosa EMG, Oliveira FDM, Guedes MVC, Monteiro ARM, Rodrigues DP, Silva LF, et al. Cuidados de enfermagem a uma puérpera fundamentados na teoria do conforto. REME Rev Min Enferm. [Internet]. 2014 [citado em 01 jan 2018]; 18(4): 845-49. doi:/10.5935/14152762.20140062

21. Krinsky R, Murillo I, Johnson J. A practical application of Katharine Kolcaba's Comfort Theory to cardiac patients. Appl Nurs Res. [Internet]. 2014 [citado em 01 jan 2018]; 27(2):147-50. doi:/10.1016/j.apnr.2014.02.004

22. Marchuk A. End-of-life care in the neonatal intensive care unit: applying comfort theory. Int J Nurs Palliat. [Internet]. 2016 [citado em 01 jan 2018]; 22(7):317-23. doi:/10.12968/ijpn.2016.22.7.317

23. Ponte KMA, Silva LF. Cuidados de enfermagem a mulheres com infarto do miocárdio: promoção do conforto sociocultural pela pesquisa-cuidado. Rev Enferm UERJ. [Internet]. 2014 [citado em 01 jan 2018]; 22(6):808-14. doi:/10.12957/reuerj.2014.15693

24. Ponte KMA, Silva LF, Aragão AEA, Guedes MVC, Zagonel IPS. Cuidado clínico de enfermagem para conforto de mulheres com IAM. Texto \& Contexto Enferm. 2014; 23(1):56-64.

25. Freire da Silva FV, Rabelo ACS, Silva LF. Processo de enfermagem no conforto do paciente com insuficiência cardíaca no domicílio. Aquichan. [Internet]. 2015 [citado em 01 jan
2018]; 15(1)116-28.

doi:/10.5294/aqui.2015.15.1.11

26. Aksoy Derya Y, Pasinlioglu T. The effect of nursing care based on comfort theory on women's postpartum comfort levels after caesarean sections. Int $\mathbf{J}$ Nurs Knowl. [Internet]. 2015 [citado em 01 jan 2018]; 28(3):138-144. doi:/10.1111/2047-3095.12122

27. Lima JVF, Guedes MVC, Silva LF, Freitas MC, Fialho AVM. Utilidade da teoria do conforto para o cuidado clínico de enfermagem à puérpera: análise crítica. Rev Gaúch Enferm. [Internet]. 2016 [citado em 01 jan 2018]; 37(4):e65022. doi:/10.1590/19831447.2016.04.65022

28. Egger-Rainer A, Trinka E, Höfler J, Dieplinger AM. Epilepsy monitoring the patients' views: a qualitative study based on Kolcaba's Comfort Theory. Epilepsy Behav. [Internet]. 2017 [citado em 01 jan 2018]; 68(n.esp):208-15. doi:/10.1016/j.yebeh.2016.11.005

29. Bergström A, Håkansson Å, Warrén Stomberg M, Bjerså K. Comfort Theory in practice-nurse anesthetists' comfort measures and interventions in a preoperative context. J Perianesth Nurs. [Internet]. 2016 [citado em 01 jan 2018]; (0):(n.esp). doi:/10.1016/j.jopan.2016.07.004

RECEBIDO: $14 / 03 / 18$

APROVADO: $16 / 07 / 18$

PUBLICADO: 07/19 